\title{
Impact of the Demand Side Management in the Planning and Operations Towards 2050
}

\author{
Ruth Domínguez \\ Miguel Carrión \\ University of Castilla-La Mancha, Department of Electrical Engineering, Toledo, Spain.
}

\begin{abstract}
In this work, we propose planning and operating models considering the uncertainty involved in the decision-making processes to: first, determine the investment decisions in generation and storage capacity to be made until 2050 to attain the CO2 emission targets in Europe, and second, analyze the market outcomes of the resulting power system. The capacity expansion model is formulated as a two-stage stochastic problem in which investment decisions in generation and storage capacity are made in the first stage considering the uncertainty related to the demand growth and the system operation represented in the second stage. A constraint of the $\mathrm{CO} 2$ emission limit is included and the influence of the level of demand side management (DSM) over the investment decisions is investigated. Moreover, the operation of the resulting European power system is analyzed by solving a two-stage stochastic problem whose first stage represents the day-ahead scheduling of the energy and reserve needs and second stage represents the real-time operation. In this model, the uncertainty related to the demand level and the available solar and wind power is considered. Consumers, generating and storage units can provide reserve capacity services. In the case study, the technical and economic operation of the European power system of 2050 is analyzed through a case study defined with historical data provided by ENTSO-E and the European Commission. The main conclusion obtained is that the DSM level has a low impact on the capacity to be built in renewable-dominated power systems if a significant storage capacity is available.
\end{abstract}

Keywords - Capacity expansion, demand side management, operation, renewables, uncertainty.

\section{Introduction}

To attain the decarbonization target of the European Commission by 2050, power systems must drastically reduce the $\mathrm{CO} 2$ emissions in the electricity production. At the same time, the electrification of the energy demands will continue growing because of the increasing use of electricity in the transportation and the heating sectors. In this sense, the so-called "Winter package" [1] published by the European Commission in May 2019 emphasizes the importance of the demand response and the consumers participation in the system operation to attain the $\mathrm{CO} 2$ emission target of 2050. Therefore, it is of high importance to analyse the impact of the demand side management (DSM) in the planning and operation of the future European power systems.

The following references study the impact of the DSM in different ways. In [2] the price of the demand elasticity as a function of the wind penetration is analised. In [3], a bi-level approach is proposed to determine the expansion decisions in distribution networks and renewable power considering the DSM and the uncertainty of the demand and the renewable production. Reference [4] presents an evaluation of the impact of the DSM on the capacity expansion of the power system in Portugal. No uncertainty is considered and the proposed model is solved using a commercial software. The authors conclude that the DSM allows to reduce the capacity to be built to reach a low carbon system. In [5], an equilibrium problem to determine the investment decisions in storage capacity considering the presence of DSM in a competitive market is presented. The results measure how much the DSM, the investment costs and the storage efficiency influence the investment decisions in storage capacity. On the other hand, [6] provides a wide analysis of the technical and economical feasibility of achieving a fully European power system. A commercial software is used to carry out the numerical analyses. Finally, [7] presents an equilibrium model with a demand response aggregator to analyse the influence of the DSM on the capacity markets. Energy, reserve capacity and investment decisions are considered. 
Considering the above, in this paper we analyse the transition of the current European power system to a new power system that meets the emissions target established for 2050 while taking into account the impact of the DSM on the investment decisions. To do this, first, we propose a capacity expansion model to determine the generation and storage capacity to be built considering the limitation of the CO2 emissions and the uncertainty of the demand growth. Second, we analyse the daily operation of the resulting power system by solving a scheduling model that dispatches simultaneously the energy and reserve capacity in the day-ahead market, considering the uncertainty of the demand level and the intermittent renewable production in the real-time operation. The proposed models are formulated using stochastic optimization. Different case studies based on real data of the European power system are solved.

\section{Methodology}

In this section, the assumptions considered to define the models are described, as well as the mathematical formulation of the capacity expansion model and the operating model.

\subsection{Assumptions}

The list with the main assumptions considered to formulate the capacity expansion and operating models follows.

- The objective of the proposed capacity expansion model is to determine the generation and storage capacity that must be built considering different levels of DSM and the uncertainty related to the evolution of the demand growh. We adopt the perspective of a system operator (SO), which seeks to minimize the total expected cost of building new capacity and supplying the demand.

- Investment decisions in both thermal and renewable generating technologies can be made. The expansion in generation and storage capacity is conditioned by three particular constraints: i) the supply of the energy demanded in each hour; ii) the procurement of minimum up and down operating reserve; and iii) a restriction on the $\mathrm{CO} 2$ emissions.

- It is important to consider the up and down operating reserve in the capacity expansion model because high reserve capacity is needed in renewable-dominated power systems to counteract the variability of the renewable power availability. All generating and storage units can provide reserve capacity. The minimum reserve requirement is determined regarding the expected value of the demand in each node, hour, day and scenario.

- We assume that a small amount of current generating capacity will be in operation until the end of the planning horizon. No existing storage capacity is considered.

- The DSM is also used to provide reserve capacity, but only a percentage of the total demand is considered as flexible demand.

- In the capacity expansion model the demand growth is considered as a random parameter. Stochastic optimization is used to introduce this fact in the decision-making process [8]. This way, a set of scenarios is generated to represent the possible realizations of the demand growth by the end of the planning horizon.

- A set of representative days of the year is selected to represent the variability of the hourly demand and available intermittent renewable power. The scenario reduction technique proposed in [9] is used to select the representative days with their weigths.

- New transmission lines cannot be built, but the limits on the power flow that can be interchanged among the nodes are included in the model. In this case, a node of the network represents a country.

- In the operating model solved afterwards to analyse the operation of the resulting power system, the random variables considered are the hourly demand and intermittent renewable power. As previously, a set of scenarios is generated to represent the possible values of those random variables along the day.

\subsection{Capacity expansion model}

The formulation of the proposed capacity expansion model is provided below. The model is formulated as a twostage stochastic optimization problem, where investment decisions are made in the first stage and the operating decisions are made in the second stage. Observe that the resulting mathematical programming problem can be formulated as a linear programming problem. Thus, the decision variables of this problem are: the generating and storage capacity to be built, the power generated by the generating units, the power charged and discharged in the storages, and the up and down reserve capacity provided by the agents. 
In the formulation given bellow, the following index are used: $n$ represents a node of the network, $g$ indicates the generating unit, $j$ and $h$ represent a day and an hour, respectively, and $s$ represents a scenario. Symbols in capital represent constants and parameters, whereas small letters represent the decision variables.

$$
\begin{aligned}
& \text { Minimize } \\
& \sum_{n \in N}\left(C_{n}^{\mathrm{BE}} e_{n}^{\mathrm{max}}+C_{n}^{\mathrm{BP}} p_{n}^{\mathrm{B}}+\sum_{g \in G} C_{g}^{\mathrm{I}} p_{g n}^{\mathrm{I}}\right)+ \\
& \sum_{n \in N} \sum_{j \in J} \sum_{h \in H} \sum_{s \in S} \pi_{s} N_{j}\left[\left(C_{n}^{\mathrm{Bup}} p_{n j h s}^{\mathrm{Bup}}+C_{n}^{\mathrm{Bdo}} p_{n j h s}^{\mathrm{Bdo}}\right)\right. \\
& \sum_{g \in G}\left(C_{g}^{\mathrm{G}} p_{g n j h s}^{\mathrm{G}}+C_{g}^{\mathrm{Gup}} p_{g n j h s}^{\mathrm{Gup}}+C_{g}^{\mathrm{Gdo}} p_{g n j h s}^{\mathrm{Gdo}}\right)+ \\
& \left.\quad\left(C_{n}^{\mathrm{Dup}} d_{n j h s}^{\mathrm{up}}+C_{n}^{\mathrm{Ddo}} d_{n j h s}^{\mathrm{do}}+C_{n}^{\mathrm{US}} d_{n j h s}^{\mathrm{US}}\right)\right]
\end{aligned}
$$

subject to

Investment decisions:

$$
\begin{aligned}
& p_{g n}^{\mathrm{I}} \leq P_{g n}^{\mathrm{Imax}}, \forall g, \forall n \\
& e_{n}^{\max } \leq E_{n}^{\mathrm{Bmax}}, \forall n \\
& p_{n}^{\mathrm{B}} \leq P_{n}^{\mathrm{Bmax}}, \forall n
\end{aligned}
$$

Emissions limitation:

$$
\sum_{g \in \Omega^{\mathrm{Th}}} \sum_{n \in N^{\mathrm{EU}}} \sum_{j \in J} \sum_{h \in H} N_{j} F_{g}^{\mathrm{CO} 2} p_{g n j h s}^{\mathrm{G}} \leq \mathrm{Max}^{\mathrm{CO} 2}, \forall s
$$

Energy balance:

$$
\begin{aligned}
& \sum_{g \in G} p_{g n j h s}^{\mathrm{GE}}+p_{n j h s}^{\mathrm{DsE}}-p_{n j h s}^{\mathrm{ChE}}-\sum_{l \mid O(l)=n} p_{l j h s}^{\mathrm{L}} \\
& +\sum_{l \mid D(l)=n} p_{l j h s}^{\mathrm{L}}=d_{n j h s}, \forall n, \forall j, \forall h, \forall s
\end{aligned}
$$

Reserve requirements:

$$
\begin{aligned}
& \sum_{g \in G} p_{g n j h s}^{\mathrm{Gup}}+p_{n j h s}^{\mathrm{Bup}}+d_{n j h s}^{\mathrm{up}} \geq R_{n j h s}^{\operatorname{minUp}}, \forall n, \forall j, \forall h, \forall s \\
& \sum_{g \in G} p_{g n j h s}^{\mathrm{Gdo}}+p_{n j h s}^{\mathrm{Bdo}}+d_{n j h s}^{\mathrm{do}} \geq R_{n j h s}^{\mathrm{minDo}}, \forall n, \forall j, \forall h, \forall s
\end{aligned}
$$

Generating units constraints:

$$
\begin{aligned}
& p_{g n j h s}^{\mathrm{Ge}}+r_{g n j h s}^{\mathrm{Gup}} \leq F_{g n j h}^{\mathrm{A}}\left(P_{g n}^{\mathrm{Ex}}+p_{g n}^{\mathrm{I}}\right), \forall g, \forall n, \forall j, \forall h, \forall s \\
& p_{g n j h s}^{\mathrm{Ge}}-r_{g n j h s}^{\mathrm{Gdo}} \geq 0, \forall g, \forall n, \forall j, \forall h, \forall s \\
& \left(p_{g n j h s}^{\mathrm{Ge}}+r_{g n j h s}^{\mathrm{Gup}}\right)-\left(p_{g n j h-1, s}^{\mathrm{Ge}}-r_{g n j h-1, s}^{\mathrm{Gdo}}\right) \\
& \quad \geq R_{g}^{\mathrm{ramp}}\left(P_{g n}^{\mathrm{Ex}}+p_{g n}^{\mathrm{I}}\right), \forall g \in \Omega^{\mathrm{Th}}, \forall n, \forall j, \forall h, \forall s \\
& \left(p_{g n j h-1, s}^{\mathrm{Ge}}-r_{g n j h-1, s}^{\mathrm{Gdo}}\right)-\left(p_{g n j h s}^{\mathrm{Ge}}+r_{g n j h s}^{\mathrm{Gup}}\right) \\
& \quad \geq R_{g}^{\mathrm{ramp}}\left(P_{g n}^{\mathrm{Ex}}+p_{g n}^{\mathrm{I}}\right), \forall g \in \Omega^{\mathrm{Th}}, \forall n, \forall j, \forall h, \forall s
\end{aligned}
$$


Demand side management:

$$
\begin{aligned}
& d_{n j h s}=\tilde{d}_{s}^{\text {growth }} D_{n j h}^{\mathrm{F}}-d_{n j h s}^{\mathrm{up}}+d_{n j h s}^{\mathrm{do}}-d_{n j h s}^{\mathrm{US}}, \\
& \forall n, \forall j, \forall h, \forall s \\
& \sum_{h \in H} d_{n j h s}^{\mathrm{up}}=\sum_{h \in H} d_{n j h s}^{\mathrm{do}}, \forall n, \forall j, \forall s \\
& 0 \leq d_{n j h s}^{\mathrm{up}} \leq \alpha \tilde{d}_{s}^{\text {growth }} D_{n j h}^{\mathrm{F}}, \forall n, \forall j, \forall h, \forall s \\
& 0 \leq d_{n j h s}^{\text {do }} \leq \alpha \tilde{d}_{s}^{\text {growth }} D_{n j h}^{\mathrm{F}}, \forall n, \forall j, \forall h, \forall s \\
& 0 \leq d_{n j h s}^{\mathrm{US}} \leq \tilde{d}_{s}^{\text {growth }} D_{n j h}^{\mathrm{F}}, \forall n, \forall j, \forall h, \forall s
\end{aligned}
$$

Storage constraints:

$$
\begin{aligned}
& p_{n j h s}^{\mathrm{Ds}}=p_{n j h s}^{\mathrm{DsE}}+p_{n j h s}^{\mathrm{Bup}}, \forall n, \forall j, \forall h, \forall s \\
& p_{n j h s}^{\mathrm{Ch}}=p_{n j h s}^{\mathrm{ChE}}+p_{n j h s}^{\mathrm{Bdo}}, \forall n, \forall j, \forall h, \forall s \\
& p_{n j h s}^{\mathrm{Ds}} \leq p_{n}^{\mathrm{B}}, \forall n, \forall j, \forall h, \forall s \\
& p_{n j h s}^{\mathrm{Ch}} \leq p_{n}^{\mathrm{B}}, \forall n, \forall j, \forall h, \forall s \\
& e_{n j h s}=e_{n j h-1, t s}-(1 / \eta) p_{n j h s}^{\mathrm{Ds}}+\eta p_{n j h s}^{\mathrm{Ch}}, \forall n, \forall j, \forall h, \forall s \\
& F_{n j}^{\min } e_{n}^{\max } \leq e_{n j h s} \leq e_{n}^{\max }, \forall n, \forall j, \forall h, \forall s \\
& e_{n j h s}=F_{n j}^{\mathrm{ini}} e_{n}^{\max }, \forall n, \forall j, h=\operatorname{Ini}(\mathrm{h}), \forall s \\
& e_{n j h s} \geq F_{n j}^{\mathrm{ini}} e_{n}^{\max }, \forall n, \forall j, h=\operatorname{Fin}(\mathrm{h}), \forall s
\end{aligned}
$$

Power exchange limitation:

$$
\begin{aligned}
& p_{l j h s}^{\mathrm{L}}=B_{l}\left(\theta_{O(l) j h s}-\theta_{D(l) j h s}\right), \forall l, \forall j, \forall h, \forall s \\
& -P_{l}^{\mathrm{Lmax}} \leq p_{l j h s}^{\mathrm{L}} \leq P_{l}^{\mathrm{Lmax}}, \forall l, \forall j, \forall h, \forall s
\end{aligned}
$$

The objective function (1) comprises two main terms: first, the investment cost of the new generating $\left(p_{g n}^{\mathrm{I}}\right)$ and energy and power storage capacities built $\left(e_{n}^{\max }, p_{n}^{\mathrm{B}}\right)$, and second, the expected cost of the up and down reserve provided by the storage units $\left(p_{n j h s}^{\text {Bup }}, p_{n j h s}^{\text {Bdo }}\right)$, the energy and up and down reserves supplied by the generating units $\left(p_{g n j h s}^{\mathrm{G}}, p_{g n j h s}^{\mathrm{Gup}}, p_{g n j h s}^{\mathrm{Gdo}}\right)$, the up and down reserves provided by the consumers $\left(d_{n j h s}^{\mathrm{up}}, d_{n j h s}^{\mathrm{do}}\right)$, and the involuntary unserved demand $\left(d_{n j h s}^{\mathrm{US}}\right)$.

Constraints (2)-(4) limit the new generation and storage capacity that can be built. Constraint (5) imposes the limit on the $\mathrm{CO} 2$ emissions from the electricity generation. Constraint (6) forces the power balance in each node, day, hour, and scenario, whereas (7) and (8) establish minimum up and down reserve capacities based on the expected value of the hourly demand.

Constraints (9)-(12) define the technical constraints of the generating units. The maximum generated power, which includes the energy to supply the demand and the up reserve, is limited to the available power in the certain hour. The generated power must be always greater than or equal to 0 even with the down reserve. Finally, (11) and (12) impose the ramping limits to the thermal power plants.

Constraints (13)-(17) characterize the demand side management. Constraint (13) defines the demand consumed $\left(d_{n j h s}\right)$ regarding the up and down reserve provided and the possible unserved demand. Constraint (14) imposes that the demand that was not supplied because of providing up reserve, must be restored within the day. Constraints (15) and (16) enforce a limit on the demand flexibililty, being parameter $\alpha$ the percentage of the flexible demand. Constraint (17) sets the limit on the involuntary unserved demand.

Constraints (18)-(25) define the technical operation of the storage units. Constraints (18) and (19) define the power discharged and charged $\left(p_{n j h s}^{\mathrm{Ds}}, p_{n j h s}^{\mathrm{Ch}}\right)$, respectively, considering the power used to complete the power balance and that allocated for the reserves. Constraints (20) and (21) limit the discharged and charged power to the power of the storage built. Constraint (22) defines the energy balance in the storage unit, (23) limits the energy capacity of the storage, while (24) and (25) set the initial level of the storage and the minimum energy level required at the end of the day, respectively.

Finally, (26) and (27) enforce the maximum power flow $\left(p_{l j h s}^{\mathrm{L}}\right)$ through the transmission lines. 


\subsection{Operating model}

The scheduling model used to analyse the daily operation of the resulting power system is based on that proposed in [10]. Thus, we consider a two-stage stochastic problem whose first stage represents the clearing of the day-ahead energy and reserve market and second stage represents the real-time operation. Therefore, the energy and reserve capacity needs are co-optimized considering the variability implicit in the real-time operation of a renewabledominated electric energy system. The variability of the real-time operation is introduced in the model through a set of scenarios that represent the possible realizations of the hourly demand and the intermittent renewable production in each hour of the day. The perspective of the $\mathrm{SO}$ is considered, hence, the goal is to minimize the total expected operating cost.

Specifically, the decision-making process consists on the following: in the first stage, generating and storage units and consumers decide how much energy to generate or consume and how much power to procure up and down reserve capacity in the day ahead of delivery; in the second stage, the supply of the demand in each hour is cleared for each scenario of the demand and the intermittent renewable power. The up and down reserves are deployed in the second stage such that the power balance is met independently of the deviations derived from the forecast errors. All the market participants, namely the generating and storage units and the consumers, can provide reserve services.

The constraints of the model comprise: the power balance in the day-ahead and real-time markets, minimum up and down reserve capacity scheduled in the day ahead, capacity and ramping limits of generating units, and the constraints characterizing the demand side management and the storage unit operation in both the day-ahead and real-time markets. Due to space limitations, the mathematical formulation is not provided, but, as previously mentioned, this model is based on that presented in [10].

\section{$3 \quad$ Numerical analysis}

The approach presented above is used to analyse the capacity expansion towards a decarbonized European power system by 2050. This section is structured as follows: first, the most relevant input data considered in the case study are described; second, the results of the capacity expansion model are shown; and third, the outcomes of the daily operation of the resulting power system are provided. To carry out the simulations we used CPLEX 12.6.1 [11] under GAMS [12] in a linux-based server of four 3.0 GHz processors and $250 \mathrm{~GB}$ of RAM.

\subsection{Input data}

The European power system is represented through a network of 24 nodes, considering each node as a country. The list of countries considered are: Austria (AT), Belgium (BE), Czech Republic (CZ), Denmark (DK), Estonia (EE), Finland (FI), France (FR), Germany (DE), Hungary (HU), Italy (IT), Latvia (LV), Lithuania (LT), Luxembourg (LU), the Netherlands (NL), Norway (NR), Poland (PL), Portugal (PT), Romania (RO), Slovakia (SK), Slovenia (SI), Spain (ES), Sweden (SE), Switzerland (CH), and United Kingdom (UK). Norway and Switzerland are not included in the constraint of the $\mathrm{CO} 2$ emission limit.

The generating technologies considered are nuclear ('Nucl'), coal ('Coal'), combined-cycle gas turbine ('CCGT'), hydropower with reservoir ('HyRe') and run-of-river ('Hrun'), onshore ('Wons') and offshore ('Woff') wind, solar photovoltaic ('SoPV'), concentrating solar power ('SCSP'), and biomass ('Biom'). The technical characteristics and the costs pertaining to each technology are provided in Table 1. We consider null the net CO2 emissions of biomass power plants. The investment and operating costs are based on the data provided by International Energy Agency [13] in their annual reports. On the other hand, the maximum capacity that can be built per technology and per country is based on the study reported in [14] considering 2050 as the target year (these data are publicly available). However, we assume: no expansion in coal because of environmental issues, no expansion in hydropower with reservoir because this resource is already exploited in Europe, and a maximum capacity of the wind and solar technologies $20 \%$ higher than the values provided in [14] for 2050. The cost of the reserve provided by the generating units is $50 \%$ higher than their operating cost. The capital recovery factor used to annualized the investment cost is computed considering an interest rate of $3 \%$ and a lifetime of the facilities of 20 years, resulting in 0.07 . 
Table 1: Characteristics of the generating technologies

\begin{tabular}{l|cc|cc} 
Technology & $R_{g}^{\mathrm{ramp}}(\mathrm{pu})$ & $F_{g}^{\mathrm{CO} 2}(\mathrm{gCO} 2 \mathrm{e} / \mathrm{kWh})$ & Inv. cost $(€ / \mathrm{kW})$ & Oper. cost $(€ / \mathrm{MWh})$ \\
\hline Nucl & 0.15 & - & 4590.0 & 10.54 \\
Coal & 0.60 & 950 & - & 18.39 \\
CCGT & 0.70 & 500 & 765.0 & 32.28 \\
HyRe & 1.00 & - & - & 20.60 \\
Hrun & 1.00 & - & 2983.5 & 3.60 \\
Wons & 1.00 & - & 1361.7 & 3.50 \\
Woff & 1.00 & - & 2945.2 & 7.50 \\
SoPV & 1.00 & - & 795.6 & 3.70 \\
SCSP & 1.00 & - & 3901.5 & 4.60 \\
Biom & 1.00 & - & 1797.7 & 15.00 \\
\hline
\end{tabular}

To determine the hourly demand and intermittent renewable production during a year, we use the historical data of 2016 available in [15], taking this year as reference. A set of 10 characteristic days is selected applying the reduction technique presented in [9] to the daily net demand (consumption minus wind and solar production). The minimum up and down reserve requirement is set to $4 \%$ of the hourly demand.

The investment costs of the storage units are $50 € / \mathrm{kWh}$ and $1000 € / \mathrm{kW}$. The maximum capacity that can be built of storage units in each node is defined as the $30 \%$ of the total demand in the most representative day of year, that in 2016 turns to be day 246. A ratio maximum energy and power capacity of 10 is considered. The cost of providing reserve is equal to $10 € / \mathrm{MWh}$. Storage units can provide up to $25 \%$ of their power capacity to the reserve service. The charging and discharging efficiency is set to $95 \%$.

The demand response cost is established to $40 € / \mathrm{MWh}$, which is comparatively higher than the cost of the generating and storage units for the same purpose. The cost of the involuntary unserved demand is $1,000 € / \mathrm{MWh}$.

Five demand growth scenarios are randomly generated taking into account the predictions made by the European Commision in [14]. The demand growth in each scenario is then defined as $18.5 \%, 20.3 \%, 25.0 \%$, $27.6 \%$, and $30.0 \%$, respectively. The scenarios are equiprobable.

The interchange capacity among countries is defined according to the data provided by ENTSO-E [15] including the capacity expansion projects already approved.

Finally, a reduction of $92 \%$ of the CO2 emissions respect to 1990 values in the aforementioned 22 European countries is imposed.

\subsection{Results: power system by 2050}

In this section, the capacity expansion results to achieve a decarbonized European power system by 2050 are shown. First, we analyse the impact of the DSM level on the new capacity built and the involuntary unserved demand in total and for each country. Second, we show the variation of the investment and operating costs for each DSM level. Third, we study the impact of the storage flexibility.

The number of variables and constraints of each problem is 3,991,969 and 3,036,270, respectively, and the execution time to solve each iteration of the problem is about 7 minutes.

The first analysis consists on solving the capacity expansion model for different levels of the demand flexibility. Four cases are compared, which differ from the percentage of flexible demand $(\alpha)$ considered, taking values from $5 \%$ to $35 \%$, respectively. Table 2 provides the total generating capacity, the share of renewable, fossil-fuel and nuclear of that capacity, the percentage of the storage energy capacity built respect to the maximum available, and the percentage of involuntary unserved demand in the 24 aformentioned countries. These aggregated results reveal the following ideas: i) the impact of the DSM level on the total generating capacity is very low; ii) the European power system globally results $75 \%$ renewable; iii) a small proportion of involuntary unserved demand is found in few countries, but it decreases with the increase of the DSM.

Table 2: Capacity expansion results for different demand response levels

\begin{tabular}{l|cccc}
\hline$\alpha$ (pu) & 0.05 & 0.15 & 0.25 & 0.35 \\
\hline Total cap. (GW) & 1602.67 & 1602.67 & 1602.67 & 1602.67 \\
\hline Renew. (\%) & 75.61 & 75.61 & 75.61 & 75.61 \\
Fossil (\%) & 19.16 & 19.16 & 19.16 & 19.16 \\
Nuclear (\%) & 5.23 & 5.23 & 5.23 & 5.23 \\
\hline Sto. (\%) & 78.16 & 77.98 & 77.98 & 77.98 \\
\hline Uns. dem (GWh) & 323.13 & 317.46 & 314.49 & 312.13 \\
Uns. dem. (\%) & 0.00 & 0.00 & 0.00 & 0.00 \\
\hline
\end{tabular}


Table 3 provides the total generating capacity installed by 2050 and the percentage of renewable units for each country and for each DSM level. The impact of the DSM level is slightly different from one country to the other. For instance, very high or high renewable power systems (renewable share higher than 80\%) as those of Spain, Portugal, Austria or Italy, do not experience a relevant impact on the total generating capacity or the share of renewables. However, in countries such as Hungary, Romania or Slovakia, there is a slightly higher variability on the share of renewable capacity with respect to the DSM level. The highest variability is found in Luxembourg.

Table 3: Total capacity (GW) and percentage of renewables per country for different DSM level

\begin{tabular}{c|cc|cc|cc|cc}
\hline & \multicolumn{2}{|c|}{$\alpha=0.05$} & \multicolumn{2}{c|}{$\alpha=0.15$} & \multicolumn{2}{c|}{$\alpha=0.25$} & \multicolumn{2}{c}{$\alpha=0.35$} \\
Country & GW & \% Ren. & GW & $\%$ Ren. & GW & $\%$ Ren. & GW & $\%$ Ren. \\
\hline PT & 23.06 & 97.43 & 23.06 & 97.43 & 23.06 & 97.43 & 23.06 & 97.43 \\
ES & 179.23 & 96.40 & 179.23 & 96.40 & 179.23 & 96.40 & 179.23 & 96.40 \\
FR & 260.96 & 68.31 & 260.96 & 68.31 & 260.96 & 68.31 & 260.96 & 68.31 \\
BE & 34.46 & 72.43 & 34.46 & 72.43 & 34.46 & 72.43 & 34.46 & 72.43 \\
LU & 5.30 & 52.04 & 5.20 & 53.09 & 3.98 & 69.26 & 2.98 & 92.60 \\
NL & 53.83 & 49.08 & 53.83 & 49.08 & 53.83 & 49.08 & 53.83 & 49.08 \\
UK & 163.20 & 70.76 & 163.20 & 70.76 & 163.20 & 70.76 & 163.20 & 70.76 \\
DE & 338.98 & 84.91 & 339.09 & 84.88 & 340.30 & 84.58 & 341.30 & 84.33 \\
CH & 35.39 & 26.73 & 35.39 & 26.73 & 35.39 & 26.73 & 35.39 & 26.73 \\
IT & 163.36 & 95.22 & 163.36 & 95.22 & 163.36 & 95.22 & 163.36 & 95.22 \\
AT & 30.16 & 97.40 & 30.16 & 97.40 & 30.16 & 97.40 & 30.16 & 97.40 \\
SI & 4.33 & 65.65 & 4.33 & 65.65 & 4.33 & 65.65 & 4.33 & 65.65 \\
CZ & 26.91 & 34.29 & 27.04 & 34.13 & 27.06 & 34.10 & 27.02 & 34.14 \\
HU & 11.22 & 34.38 & 11.35 & 33.97 & 11.31 & 34.10 & 12.27 & 31.43 \\
RO & 31.75 & 77.92 & 31.80 & 77.82 & 31.87 & 77.65 & 30.86 & 80.17 \\
SK & 9.67 & 44.14 & 9.23 & 46.26 & 9.16 & 46.59 & 9.27 & 46.04 \\
DK & 21.64 & 84.95 & 21.64 & 84.95 & 21.64 & 84.95 & 21.64 & 84.95 \\
PL & 74.22 & 47.12 & 74.36 & 47.03 & 74.38 & 47.02 & 74.34 & 47.04 \\
NR & 44.06 & 64.59 & 44.06 & 64.59 & 44.06 & 64.59 & 44.06 & 64.59 \\
SE & 57.91 & 65.46 & 57.91 & 65.46 & 57.91 & 65.46 & 57.91 & 65.46 \\
FI & 21.43 & 59.85 & 21.43 & 59.85 & 21.43 & 59.85 & 21.43 & 59.85 \\
EE & 3.15 & 96.58 & 3.15 & 96.58 & 3.15 & 96.58 & 3.15 & 96.58 \\
LV & 3.69 & 78.16 & 3.69 & 78.16 & 3.69 & 78.16 & 3.69 & 78.16 \\
LT & 4.75 & 64.71 & 4.75 & 64.71 & 4.75 & 64.71 & 4.75 & 64.71 \\
\hline EU & 1602.67 & 75.61 & 1602.67 & 75.61 & 1602.67 & 75.61 & 1602.67 & 75.61 \\
\hline
\end{tabular}

Table 4: Costs for different demand response level (bill. €)

\begin{tabular}{c|ccc|c|c|c|c}
\hline$\alpha$ & Inv. & Oper. & Unserv. & Total & Res. Bat. & DSM & Gen. Unit \\
\hline 0.05 & $1,609.5$ & $2,228.1$ & $8,596.7$ & $10,932.6$ & $1,986.9$ & 136.5 & 104.7 \\
0.15 & $1,609.4$ & $2,380.3$ & $8,380.1$ & $10,868.2$ & $1,987.0$ & 288.7 & 104.6 \\
0.25 & $1,609.4$ & $2,447.2$ & $8,278.4$ & $10,833.4$ & $1,987.1$ & 355.5 & 104.6 \\
0.35 & $1,609.4$ & $2,471.1$ & $8,240.1$ & $10,819.0$ & $1,987.1$ & 379.4 & 104.6 \\
\hline
\end{tabular}

The second type of results comprise the investment and operating costs in the whole system for each DSM level. Specifically, Table 4 shows: the annualized investment cost (Inv.), the total expected operating cost (Oper.), the expected penalization cost of the involuntary unserved demand (Unserv.), the total expected cost, i.e., the objective function value, the expected cost associated to the reserve provision from the storage units (Res. Bat.), the expected cost of the DSM, and the generating unit cost of providing energy and reserve capacity. Looking at the results of Tables 2-4, we can conclude that the DSM has a noticeable impact on the system operation, whereas it is not relevant for the investment decisions. Moreover, the third column of Table 4 indicates that the operating cost of the system increases with the DSM level and this increment is linked to the DSM cost (seventh column), since neither the reserve provision cost of the storage units nor the generating unit costs vary. Note that the involuntary unserved demand is highly penalized.

To end this section, the impact of the storage capacity in the system is analysed. In particular, we solve two additional cases: first, no storage capacity is considered, and second, the reserve capability of storage units is increased up to $50 \%$ of their power capacity. In both cases we assume a DSM level of $35 \%$. The main results of the two cases are provided in Table 5, namely the total generating capacity by 2050, the percentage of renewable capacity, the involuntary unserved demand, the annualized investment cost and the expected cost of the DSM. 
The presence or not of storage capacity has a crucial impact on the planning and system operation. The high participation of the DSM in the no-storage case cannot avoid a large amount of unserved demand. However, having very flexible storage capacity allows a better integration of the renewable production and, hence, the possibility of supplying the whole demand.

Table 5: Impact of the storage capacity

\begin{tabular}{c|c|c|c|c|c}
\hline Case & Total capacity (GW) & \% Renewable & Uns. Dem. (GWh) & Inv. Cost (bill. $€$ ) & DSM cost (bill. $€$ ) \\
\hline No sto. & $1,517.3$ & 79.8 & $5,792.1$ & $1,479.1$ & $10,717.7$ \\
50\% Res. Bat. & $1,564.2$ & 77.5 & 0.0 & $1,579.3$ & 0.0 \\
\hline
\end{tabular}

\subsection{Results: operation of the 2050 power system}

A summary of the operating outcomes of the 2050 power system is provided in this section. Recall that in the operating model the day-ahead energy and reserves schedule is co-optimized considering the uncertainty in the real-time operation. The DSM level considered is $35 \%$ with a cost of $20 € / \mathrm{MWh}$, while the demand growth respect to 2016 values is set to $25 \%$. Since the influence of the storage capacity over the DSM is very high, we compare the outcomes obtained from three cases that differ on the reserve capacity provided by the storage units. In particular, we consider a reserve capacity of the storage units (Res. cap. storage) equal to $2 \%, 20 \%$ and $60 \%$ of their power capacity. To represent the yearly variability 25 representative were selected. Table 6 provides the following aggregated results for each case: the share of the renewable production respect to the total (Ren. prod.), the DSM in the real-time operation, the expected total $\mathrm{CO} 2$ emissions (CO2 emi.), and the expected operating cost.

Table 6: Summary of the operating outcomes

\begin{tabular}{c|c|c|c|c}
\hline $\begin{array}{c}\text { Res. cap. } \\
\text { storage }\end{array}$ & $\begin{array}{c}\text { Ren. prod. } \\
(\%)\end{array}$ & $\begin{array}{c}\text { DSM } \\
(\text { TWh })\end{array}$ & $\begin{array}{c}\text { CO2 emi. } \\
\text { (mill. ton) }\end{array}$ & $\begin{array}{c}\text { Cost } \\
\text { (bill. } €)\end{array}$ \\
\hline $2 \%$ & 68.8 & 6224 & 380 & 4227 \\
$20 \%$ & 69.1 & 1723 & 380 & 4137 \\
$60 \%$ & 69.1 & 1623 & 372 & 4134 \\
\hline
\end{tabular}

Tables 7-9 provide the percentage of renewable production (Ren. prod.), the DSM provided by the consumers in the real-time operation, and the expected CO2 emissions in each country.

\section{Conclusions}

Attaining a decarbonized European power system will require the participation of all agents in the system operation, but the participation of each agent will strongly depend on their operating costs and their flexibility. A more detailed analysis will follow this research work to go into the detail of the results provided in this paper. 
Table 7: Operating outcomes in the case with $2 \%$ of storage reserve capacity

\begin{tabular}{c|c|c|c}
\hline Country & Ren. prod. (\%) & DSM (TWh) & CO2 emi. (mill. ton) \\
\hline AT & 99.4 & 147.8 & 0.38 \\
BE & 54.2 & 118.9 & 21.12 \\
CH & 51.2 & 578.3 & 0.03 \\
CZ & 19.0 & 200.0 & 18.10 \\
DE & 71.0 & 619.8 & 162.05 \\
DK & 99.5 & 44.7 & 0.23 \\
EE & 94.7 & 24.0 & 0.26 \\
ES & 99.9 & 456.4 & 0.35 \\
FI & 68.2 & 56.6 & 3.91 \\
FR & 50.3 & 628.9 & 19.48 \\
HU & 27.0 & 188.4 & 1.11 \\
IT & 97.8 & 868.6 & 6.59 \\
LT & 42.2 & 5.1 & 0.00 \\
LU & 90.1 & 7.3 & 0.16 \\
LV & 78.3 & 13.3 & 0.80 \\
NL & 42.7 & 170.7 & 55.20 \\
NR & 100.0 & 999.3 & 0.03 \\
PL & 35.2 & 76.7 & 67.20 \\
PT & 100.0 & 20.2 & 0.00 \\
RO & 64.3 & 13.7 & 4.74 \\
SE & 51.5 & 58.4 & 1.16 \\
SI & 37.7 & 36.7 & 1.60 \\
SK & 40.5 & 29.0 & 6.01 \\
UK & 75.1 & 861.2 & 9.41 \\
\hline
\end{tabular}

Table 8: Operating outcomes in the case with $20 \%$ of storage reserve capacity

\begin{tabular}{c|c|c|c}
\hline Country & Ren. prod. (\%) & DSM (TWh) & CO2 emi. (mill. ton) \\
\hline AT & 99.4 & 4.7 & 0.40 \\
BE & 55.2 & 0.0 & 20.57 \\
CH & 49.5 & 0.0 & 0.03 \\
CZ & 19.3 & 0.0 & 17.75 \\
DE & 71.1 & 0.0 & 160.81 \\
DK & 99.3 & 0.0 & 0.26 \\
EE & 95.2 & 0.0 & 0.24 \\
ES & 99.9 & 893.9 & 0.35 \\
FI & 68.8 & 0.0 & 3.44 \\
FR & 50.9 & 0.0 & 17.73 \\
HU & 27.4 & 0.0 & 1.11 \\
IT & 97.9 & 663.7 & 6.32 \\
LT & 43.0 & 0.0 & 0.00 \\
LU & 84.4 & 0.0 & 0.28 \\
LV & 80.4 & 0.0 & 0.72 \\
NL & 43.0 & 0.0 & 55.27 \\
NR & 99.9 & 82.4 & 0.05 \\
PL & 36.5 & 0.0 & 63.77 \\
PT & 100.0 & 10.5 & 0.00 \\
RO & 65.2 & 0.0 & 4.23 \\
SE & 51.4 & 0.0 & 1.11 \\
SI & 38.0 & 0.0 & 1.57 \\
SK & 41.4 & 0.0 & 5.97 \\
UK & 74.8 & 68.3 & 9.75 \\
\hline
\end{tabular}


Table 9: Operating outcomes in the case with $60 \%$ of storage reserve capacity

\begin{tabular}{c|c|c|c}
\hline Country & Ren. prod. (\%) & DSM (TWh) & CO2 emi. (mill. ton-eq) \\
\hline AT & 99.3 & 4.3 & 0.41 \\
BE & 55.4 & 0.0 & 20.46 \\
CH & 49.6 & 0.0 & 0.03 \\
CZ & 19.3 & 0.0 & 17.76 \\
DE & 71.0 & 0.0 & 160.80 \\
DK & 99.4 & 0.0 & 0.25 \\
EE & 95.2 & 0.0 & 0.24 \\
ES & 99.9 & 874.6 & 0.36 \\
FI & 68.7 & 0.0 & 3.47 \\
FR & 50.9 & 0.0 & 17.75 \\
HU & 27.4 & 0.0 & 1.09 \\
IT & 97.9 & 671.8 & 6.33 \\
LT & 43.1 & 0.0 & 0.00 \\
LU & 81.9 & 0.0 & 0.33 \\
LV & 80.4 & 0.0 & 0.72 \\
NL & 43.0 & 0.0 & 55.23 \\
NR & 99.9 & 0.0 & 0.05 \\
PL & 36.5 & 0.0 & 63.80 \\
PT & 100.0 & 11.2 & 0.01 \\
RO & 65.2 & 0.0 & 4.24 \\
SE & 51.4 & 0.0 & 1.12 \\
SI & 38.0 & 0.0 & 1.57 \\
SK & 41.4 & 0.0 & 5.98 \\
UK & 74.6 & 65.1 & 9.78 \\
\hline
\end{tabular}




\section{References}

[1] European Commission, "Clean energy for all Europeans", Publications Office of the European Union, Luxembourg, 2019. doi:10.2833/9937

[2] S. Behboodi, D. P. Chassin, C. Crawford, and N. Djilali, "Renewable resources portfolio optimization in the presence of demand response", Applied Energy, vol. 162, pp. 139-148, 2016.

[3] M. Asensio, G. Muñoz-Delgado, and J. Contreras, "Bi-Level Approach to Distribution Network and Renewable Energy Expansion Planning Considering Demand Response", IEEE Trans. Power Syst., vol. 32, no. 6, pp. 4298-4309, 2017.

[4] J. Anjo, D. Neves, C. Silva, A. Shivakumar, and M. Howells, "Modeling the long-term impact of demand response in energy planning: The Portuguese electric system case study", Energy, vol. 165, pp. 456-468, 2018.

[5] Y. Dvorkin, "Can Merchant Demand Response Affect Investments in Merchant Energy Storage?", IEEE Trans. Power Syst., vol. 33, no. 3, pp. 2671-2683, 2018.

[6] W. Zappa, M. Junginger, and M. van den Broek, "Is a 100\% renewable European power system feasible by 2050?", Applied Energy, vol. 233-234, pp. 1027-1050, 2019.

[7] M. Á. Lyncha, S. Nolanc, M. T. Devinea, M. OMalley, "The impacts of demand response participation in capacity markets", Applied Energy, vol. 250, pp. 444-451, 2019.

[8] J. R. Birge and F. Louveaux, "Introduction to Stochastic Programming", Springer-Verlag, New York, NY, 1997.

[9] N. Growe-Kuska, H. Heitsch, and W. Romisch, "Scenario reduction and scenario tree construction for power management problems", in Proc. IEEE Bologna Power Technol. Conf., Bologna, Italy, Jun. 2003.

[10] R. Domínguez, A. J. Conejo and M. Carrión, "Operation of a fully renewable electric energy system with CSP plants", Applied Energy, vol. 119, pp. 417-430, 2014.

[11] R. E. Rosenthal, "GAMS, A User's Guide". Washington, DC: GAMS Development Corp.; 2015.

[12] The ILOG CPLEX; 2015. [Online]. Available: http://www.ilog.com/products/cplex/.

[13] International Energy Agency (IEA), "World Energy Outlook 2016", 2018. Available: https://www. iea.org/weo/

[14] European Commission. "EU Reference Scenario 2016 (2015). Energy, transport and GHG emissions. Trends to 2050". Available: https://ec.europa.eu/energy/node/186

[15] ENTSO-E transparency platform, 2018. Available: https://transparency.entsoe.eu/ 